\title{
Computational models of mentalizing
}

\author{
Bryan Gonzalez \& Luke J. Chang* \\ Department of Psychological and Brain Sciences \\ Dartmouth College \\ Hanover, NH, 03755
}

${ }^{*}$ Corresponding author (luke.j.chang@dartmouth.edu)

Word count: 5,055

Keywords: mentalizing, theory of mind, game theory, psychological game theory, reinforcement learning, bayesian inference 


\begin{abstract}
Humans have a remarkable ability to infer and represent others' mental states such as their beliefs, goals, desires, intentions, and feelings. In this chapter, we review some of the innovations that have developed in economics, computer science, and cognitive neuroscience in modeling the computations underlying several mentalizing operations. Broadly, this involves building models of how agents infer the mental states of other agents within constrained environments. These models include modules for: representing the goals and desires of an agent (e.g., maximize reward, or minimize embarrassment), inferring the mental states of other agents (e.g., beliefs, goals, desires, intentions, and feelings), and integrating these goals and mentalizing computations to produce optimal behavioral policies to navigate the environment. The mathematical operationalization of these constructs provides a general framework that can be validated by behavior and neural recording techniques and extended in the future by contributions from multiple scientific disciplines.
\end{abstract}




\section{Introduction}

Humans have an incredible ability to effortlessly infer another's mental state. What type of computations facilitate this ability? One of the pioneers of social psychology, Kurt Lewin, attempted to develop a formal psychological framework using mathematical tools such as topology and vector calculus (Lewin, 1936, 1938). He posited that behavioral actions, $B$, can be described as a function of an individual person, $P$, acting within an environment, $E$, $B=f(P, E)$ (Lewin, 1936). An individual's goals and mental states will influence their behavior, but the possible trajectories will ultimately be constrained by the environment. This framework may be useful in understanding how we can predict another person's behavior. Mentalizing describes the psychological process of inferring another person's beliefs, intentions, desires, and feelings. These inferences will likely be influenced by the environment (e.g., what would a reasonable person do in this situation?), as well as the specific actions of the person (e.g., they are heading straight to the food, they must be hungry). Since the early days of Lewin, there have been many advances in formalizing the computations of mentalizing. In this chapter, we will provide a brief overview of some of the key concepts and recent developments.

How are we able to effortlessly infer others' beliefs, intentions, desires, and feelings? Information cues about these hidden states can be perceived from many sensory modalities. For example, subtle facial expressions can communicate information about an individual's internal state (Darwin, 1886). However, these signals are noisy and do not necessarily directly correspond to specific internal states. As an example, consider how we might infer if another person is enjoying their meal. After taking a bite of specific dish, the person smiles. If smiling was completely involuntary and only occurred when enjoying an experience, then this should lead us to infer that the person is enjoying the food. However, perhaps the person knows that we personally prepared the dish and is smiling to politely indicate acknowledgment of our efforts. Multiple internal states can produce the same facial expression. Early neurophysiologists demonstrated that there are distinct neural pathways that independently innervate voluntary and involuntary control of facial expressions such as smiles (Duchenne de Boulogne, 1876; Müri, 2016). Yet, we are able to reliably distinguish voluntary produced, from spontaneously induced smiles, and can also interpret the internal states of others from their prosody of speech (Achim, Guitton, Jackson, Boutin, \& Monetta, 2013; Baltaxe, 1991), body posture (Parkinson, Walker, Memmi, \& Wheatley, 2017), or their complex volitional actions. This sensitivity to the social environment is so acute that we sometimes even interpret non-human objects in the external world as possessing volitional agency with their own internal feelings, beliefs and desires (Heider \& Simmel, 1944). Thus, inferring the meaning behind these social behaviors requires reasoning about the potential latent mental states that cause the observed behavior.

Empirical experiments have been instrumental in improving our understanding of the process of mentalizing beyond philosophical thought experiments. Philosopher Daniel Dennet first 
proposed a stringent test for the presence of theory-of-mind (ToM) - the prediction of another person's behavior on the basis of a person's false belief (Dennett, 1978). In his view, a true belief would be insufficient because it would be impossible to discern whether a person behaves in accordance with reality or in accordance with their own beliefs about reality. The, 'Sally-Ann false-belief test' was designed to meet this challenge (Wimmer \& Perner, 1983) and has been well studied across a wide range of ages (Wellman, Cross, \& Watson, 2001), species (Call \& Tomasello, 1999; Krupenye, Kano, Hirata, Call, \& Tomasello, 2016), and special populations (Baron-Cohen, Leslie, \& Frith, 1985; Rosenthal, Hutcherson, Adolphs, \& Stanley, 2019). More recently the neural basis of mentalizing has been studied when reading stories involving mental states (Saxe \& Kanwisher, 2003/8), making inferences about others' preferences (Jenkins, Macrae, \& Mitchell, 2008; Mitchell, Heatherton, \& Macrae, 2002), watching movies that requiring inferring characters' mental states (Pantelis, Byrge, Tyszka, Adolphs, \& Kennedy, 2015; Richardson, Lisandrelli, Riobueno-Naylor, \& Saxe, 2018), and playing interactive games that require reasoning about other's intentions (Chang, Smith, Dufwenberg, \& Sanfey, 2011; Hampton, Bossaerts, \& O’Doherty, 2008; Sul, Güroğlu, Crone, \& Chang, 2017). These studies have identified a network of brain regions that appear to be reliably involved in mentalizing including the dorsomedial prefrontal cortex (dmPFC), temporal parietal junction (TPJ), superior temporal sulcus (STS), ventromedial prefrontal cortex (vmPFC), and posterior cingulate cortex (PCC) (Amodio \& Frith, 2006; Blakemore, 2008; Frith \& Frith, 2006; Van Overwalle, 2009).

While clever experiments have been instrumental in identifying the bounds of mentalizing, we still know very little about how these mental operations are specifically computed. A collection of empirical findings testing simple hypotheses are unlikely to reveal the complex processes underlying how people are able to infer the latent causes underlying others' behavior (Lewin, 1936; Newell, 2014). Instead, we might consider following trends in cognitive science and developing comprehensive and constrained models of cognitive architectures that are capable of predicting behavior across a range of different tasks (Newell, 1994; O'Reilly, Hazy, \& Herd, 2016). Computational models provide a way to formally operationalize the mental processes that are hypothesized to underlie a specific cognitive operation (Jolly \& Chang, 2019). This allows us to simulate behavior in various tasks and assess if the model can accurately account for how people behave in these environments. In addition, model representations of specific mental processes can be combined with neural recording techniques to identify the underlying neural circuitry, which can open up new avenues for improving our understanding of mentalizing (Cheong, Jolly, Sul, \& Chang, 2017).

In this chapter, we will explore the burgeoning use of computational models to study mentalizing. The mathematical operationalization of these abilities provides a common language for researchers from disparate disciplines to bring together diverse perspectives (Jolly \& Chang, 2019). In support of this interdisciplinary endeavor, we will briefly review some of the contributions to modeling mentalizing from the disciplines of cognitive science, computer science, economics, and cognitive neuroscience. 


\section{Game Theory of Mind}

The ability to understand the minds of others has been modeled by economists over the past 70 years in the context of game theory (von Neumann \& Morgenstern, 2007). Analogous to Lewin's proposition to model behavior as a function of the person and environment, game theory also models players and the structure of the game environment. Games refer to the mathematical descriptions of the strategies available to the players and of the payoffs resulting from those strategies. Additional details may include the sequence of play, the actions available to each player at each stage of the game, and the information available to each player. Players' beliefs are represented via probability distributions over actions, states, or other players' beliefs. Games involving two or more players and can be competitive or cooperative and played once (single shot) or repeatedly. One nice attribute of game theoretic modeling is that the behaviors of each player can be predicted without ever directly observing behavior. These predictions are referred to as solution concepts and often involve an equilibrium concept such as a Nash Equilibrium in which each player is assumed to know the strategy of the other players and no player can improve their payoff by changing their strategy (Nash, 1950). In this section, we will briefly discuss how the topics of strategic reasoning and psychological game theory have provided innovations in modeling mentalizing computations.

\section{Strategic Reasoning}

In economic games, players form strategies about which actions to take in the game. Players are assumed to be utility maximizers and will select the action that leads to the highest payoff. However, the payoffs in games are also determined by other players' actions. Thus, maximizing payoffs requires engaging in strategic reasoning, which may involve selecting an action based on first-order beliefs about the likelihood of other players taking a specific action. Some players may engage in even higher levels of strategic reasoning and form beliefs about other players' beliefs about their actions, which are referred to as second-order beliefs. Theoretically, there could be an infinite recursion of beliefs about beliefs. However, our brains do not possess infinite computational resources and instead there appear to be bounds on our cognitive capacity (Simon, 1956) and level of recursive reasoning or strategic sophistication (C. F. Camerer, Ho, \& Chong, 2015; C. Camerer, Ho, \& Chong, 2003; Stahl \& Wilson, 1995; Yoshida, Dolan, \& Friston, 2008).

The concept of bounded rationality in recursive mental state inference can be illustrated in the beauty contest game (Nagel, 1998). In this game, players simultaneously choose a number between 0 and 100, and are informed that the guess closest to $2 / 3$ of the average number wins a fixed prize. Players use a set of recursive thinking steps to choose the strategy expected to maximize payoffs given their beliefs about the sophistication of other players. Zero-step players do not employ any strategic reasoning and choose their numbers randomly according to a uniform distribution. More sophisticated one-step players believe they are playing against 
zero-step players. The average of zero-step players choices will be approximately 50 , and thus will choose 33 (i.e., 2/3 of 50). Two-step players believe they are playing against a mixture zero and one-step players and reason about the proportions of each. In this game, payoff is maximized by adopting a strategy that is one level of sophistication higher than the average of all other players. If players can employ an infinite level of strategic sophistication, the Nash equilibrium in this game is to choose 0 . In practice, behavior in the game can be approximated using a Poisson distribution with a $\lambda=1.8$, which means that the distribution of sophistication levels is approximately: zero $17 \%$, one $30 \%$, two $27 \%$, and three $16 \%$ (C. Camerer et al., 2003). In other words, most players use a level 1 or 2 sophistication in their strategic reasoning. Using higher levels of sophistication in the beauty contest (Coricelli \& Nagel, 2009) and other games (Yoshida, Seymour, Friston, \& Dolan, 2010) has been shown to recruit increased activation in dmPFC when reasoning about other players, which is consistent with the interpretation that strategic reasoning involves representing others mental states.

\section{Psychological Game Theory}

Modeling behavior in games does not only involve considering strategic reasoning, but also the goals and motivations of the players. Classic economic theory assumes that people receive utility solely from material payoffs. However, people have many other motivations that can influence behavior, such as reputational concerns and emotions (e.g., guilt, disappointment, regret, frustration, anger, etc), which are more abstract and difficult to quantify. Psychological Game Theory (PGT) is a framework to model motivations that depend on one's own or others' beliefs (Battigalli \& Dufwenberg, 2009; Battigalli, Dufwenberg, \& Others, 2019; Geanakoplos, Pearce, \& Stacchetti, 1989). This framework has been useful in providing formal operationalizations of how emotions such as guilt (Battigalli \& Dufwenberg, 2007; Chang et al., 2011; van Baar, Chang, \& Sanfey, 2019) and anger (Battigalli, Dufwenberg, \& Smith, 2015; Chang \& Sanfey, 2013) can be represented as belief-dependent psychological payoffs in a utility function and can accurately predict behavior in a variety of cooperative games (Chang \& Smith, 2015). For example, guilt can be modeled as a motivation to avoid disappointing a relationship partner by taking an action that is consistent with a player's second order beliefs about what they believe their partner expects them to do. When playing games in the scanner, these belief-dependent emotions have been linked to regions of the brain that are involved in processing negative affect and error monitoring such as the anterior insula and anterior cingulate cortex (ACC) (Chang \& Sanfey, 2013; Chang et al., 2011; van Baar et al., 2019).

In addition to modeling psychological payoffs associated with specific emotions, PGT has also been used to model how player's perceive another player's intentions behind their actions. For example, Rabin (1993) proposed a model of intention-based reciprocity where kind intentions are reciprocated with kind actions, while unkind intentions are reciprocated with unkind actions. Though, this tit-for-tat strategy had been previously described (Akerlof, 1982; Goranson \& Berkowitz, 1966; Trivers, 1971), this was the first attempt to formally model the mentalizing process of inferring the hidden intentions of the other player from behavior in the 
game. This model was found to be a better explanation of behavior than the popular other-regarding preference model of inequity aversion (Bolton \& Ockenfels, 2000; Fehr \& Schmidt, 1999) in a clever experimental design of mini-ultimatum games (Falk, Fehr, \& Fischbacher, 2003). In this study, participants received a proposal of a $80-20 \%$ split of a pot of money and were asked to decide whether to accept the offer, in which they would receive the $20 \%$ portion, or reject it, in which case neither player would receive any money. The inequity-aversion model predicts that participants should reject the offer because that results in a more equitable outcome (\$0 - \$0) than the 80-20 proposal. The experimenters provided an interesting twist by manipulating information about the counterfactual option that the proposer could have alternatively chosen. Most participants decided to reject the proposal when the 80-20 was chosen over a 50-50 split, but chose to accept the same proposal when it was selected over a 90-10 split. Participants presumably inferred that the proposer had good intentions and accepting the offer would acknowledge and reciprocate this intention, while more selfish intentions of not selecting a 50-50 split would be punished. These types of other-regarding preferences are unlikely to be a stable preference that is inherent to a specific individual as they appear to evolve over the course of development. Children between the ages of 3-8 switch from being purely selfish to developing preferences for inequity-aversion (Fehr, Bernhard, \& Rockenbach, 2008). Preferences to consider others intentions appears to shift later in adolescence, which is mediated by cortical maturation in the dmPFC (Sul et al., 2017).

Game theory has provided a unique opportunity to model mentalizing processes of representing others beliefs, intentions, feelings, and desires in the context of constrained interactive economic games. Because these games are relatively simple, they are well suited for neuroimaging environments (Chang \& Sanfey, 2013; Chang et al., 2011; Coricelli \& Nagel, 2009; Hampton et al., 2008; King-Casas et al., 2005; McCabe \& Smith, 2000; Sanfey, Rilling, Aronson, Nystrom, \& Cohen, 2003; Sul et al., 2017; Yoshida et al., 2010). Furthermore, Because these models represent different types of mentalizing operations, model-based fMRI analytic strategies (J. O'Doherty, Hampton, \& Kim, 2007) have been fruitful in mapping these computations to brain activity in many regions known to be involved in representing others mental states.

\section{Machine Theory of Mind}

How does a person come to intuitively know that a certain look from their partner means they had a tough day at work? Why can best friends have entire dialogues with each other without completing a single sentence? These impressive feats of mentalizing are a result of our impressive ability to continually learn statistical regularities from our experiences as we navigate the social world. In this section, we will explore how machine learning techniques have been used to model human learning about the beliefs, goals, and desires of others. One subdomain of machine learning that has shown promise in modeling artificial intelligence is reinforcement-learning (RL). Similar to Lewin's model of behavior as a function of a person and environment, $\mathrm{RL}$ models agents acting within an abstracted environment. These types of 
models have witnessed extraordinary advances in recent years, merging supervised learning techniques such as deep neural network architectures to develop agents that outperform human experts in complicated strategic games, such as backgammon (Tesauro, 1994), chess (Campbell, Hoane, \& Hsu, 2002), Atari (Mnih et al., 2015), go (Silver et al., 2018), and poker (Brown \& Sandholm, 2019; Moravčík et al., 2017). While these models have achieved impressive performance that is beginning to generalize across tasks, they require considerable amounts of data and computational power, and it is unclear if the models are developing strategies that can help us understand how humans actually perform these computations. We will focus our discussion on some of the basic concepts from reinforcement-learning to build an intuition for how these algorithms might help us model how people reason about others. We will provide examples in which people appear to use prediction errors to learn about others, their experiences, and optimal actions. We will also discuss how inverse-reinforcement learning can be used to understand how we make predictions about another person's hidden mental state based on our observation of their actions.

\section{Estimating Value}

Formal reinforcement learning models were first inspired by learning experiments in psychology (Rescorla, Wagner, \& Others, 1972) and were subsequently advanced by computer scientists (Sutton \& Barto, 1998). RL models provide a computational approach to understanding and automating goal-directed learning and decision-making. In this framework, agents learn actions within an environment that maximize their overall cumulative reward. Predictions of future value $V$ at timepoint $t+1$ can be iteratively learned via prediction error, which is simply the difference between our experienced and expected rewards.

$$
V(t+1) \leftarrow V(t)+\alpha[r-V(t)]
$$

The difference between the expected value $V$ at time $t$ and the experienced reward $r$ constitutes an error, which can be reduced by taking a step towards the "target" (Sutton \& Barto, 2018). We can think of the target as some true desired outcome presumed to come directly from the environment. An RL agent seeks an improved estimate of a signal's value by incrementally updating its old estimate, via a step size $\alpha$, based on experienced errors. This provides a way to learn the value of a given signal in the environment via trial-and-error (Pearce \& Hall, 1980; Rescorla et al., 1972). This simple prediction-error learning signal is reliably associated with activity in the ventral striatum when learning in simple tasks (Bartra, McGuire, \& Kable, 2013; McClure, Berns, \& Montague, 2003; J. P. O’Doherty, Dayan, Friston, Critchley, \& Dolan, 2003). Interestingly, this same learning system appears to aid in learning about an individual's moral character from repeated interactions in economic games such as trustworthiness (Chang, Doll, van 't Wout, Frank, \& Sanfey, 2010; Delgado, Frank, \& Phelps, 2005; Fareri, Chang, \& Delgado, 2012). 


\section{Observational Reinforcement Learning}

$R L$ provides a framework to not only learn about the moral character of another person, but also vicariously about the world via another person's experience. While observing an agent interact with their environment, prediction errors about the outcome can be computed for the agent rather than the observer. Vicarious reinforcement learning allows an observer to develop beliefs about the world without ever directly experiencing any outcomes (Lindström, Golkar, Jangard, Tobler, \& Olsson, 2019; Selbing \& Olsson, 2017). These observed outcome prediction errors have been shown to be correlated with activity in the dorsal striatum and vmPFC (Burke, Tobler, Baddeley, \& Schultz, 2010; Hill, Boorman, \& Fried, 2016; Suzuki et al., 2012).

Sometimes, however, an observer can learn to imitate which actions to take, even when the outcomes or rewards for an observed agent's actions are not directly observable. Rather than learning the reward contingencies of the environment, imitation learning involves learning to take a particular action based on the extent to which another agent was observed to take that action in the past. Here, the value of a given action is computed through positive reinforcement if the action was performed by an observed agent, while unchosen actions are negatively reinforced. The difference between the action performed by an observed agent and the action that was expected by the observer constitute 'action prediction errors' and can provide a learning signal similarly to observed reward prediction errors. In contrast to vicarious learning, imitation learning signals have been associated with a different set of brain regions including the dmPFC, dorsolateral prefrontal cortex (dIPFC) (Burke et al., 2010), and the inferior parietal lobule (Suzuki et al., 2012), - a candidate location of mirror neurons in humans (Chong, Cunnington, Williams, Kanwisher, \& Mattingley, 2008).

\section{Inverse Reinforcement Learning}

One potential drawback of both vicarious and imitation learning is the assumption that an observer possesses the same value function as the agent. However, there are many situations where two or more agents place vastly different values on the same thing. For instance, how do we learn about another person's food preferences after repeated dining experiences (Jern, Lucas, \& Kemp, 2017)? To solve this, computational models of observational learning must permit inferences about another agent's goals independent of the observer's own preferences or value function. One way this could occur is by combining prior information with observed evidence of a given goal in order to update an observer's beliefs about an agent's goal in a Bayesian manner. Unlike standard RL, which attempts to learn the optimal actions given a reward function, inverse reinforcement learning, attempts to recover the learned reward function for which an observed agent's actions would be optimal (Abbeel \& Ng, 2004; Ng, Russell, \& Others, 2000). This type of algorithm is particularly well suited to model hidden beliefs, goals, and desires from observing others' actions (Baker, Jara-Ettinger, Saxe, \& Tenenbaum, 2017; Jara-Ettinger, 2019; Jern et al., 2017; Koster-Hale \& Saxe, 2013). 
Inverse RL was recently examined in a study where observers were tasked with choosing between slot machines that would yield different types of food (Collette, Pauli, Bossaerts, \& O'Doherty, 2017). Observers were only able to learn indirectly about the types of foods that were paid out through each of the slot machines by observing two other agent's actions, but not the outcomes of the agents' decisions. Importantly, observers knew that they shared food preferences with only one of the agents. The authors found that an inverse RL model explained participants' choices better than imitation learning. Consistent with previous studies (Burke et al., 2010), they found dmPFC activity to be involved in encoding the agent's (not the observer's) expected value of an action at the moment participants observed the agent chose that action. Because the agent's represented preferences differed from their own, the authors suggest that observers may have been simulating 'what it would be like' to be the agent. Common mentalizing regions such as the TPJ and STS were also shown to track the degree to which observers updated their knowledge of the environmental reward structure. In addition to the mentalizing network, the dorsal striatum, lateral PFC, and pre-sensory-motor area tracked these updates on a trial by trial basis, suggesting that observational learning about reward distributions through inverse $\mathrm{RL}$ depends on regions commonly recruited for mentalizing, as well as areas generally recruited for experiential learning.

\section{Bayesian Theory of Mind}

A fundamental assumption made by game theoretic and RL models is that the ultimate goal of observed behavior is the maximization of rewards gained from one's environment. When an agent is faced with a decision about which action to take, they do so by intuiting a form of a cost function that, for each potential action in a given state, provides information to the agent of the resources (effort, time, money, etc.) required to perform it. The costs of taking an action are considered along with the agent's reward function, which maps environmental states to intrinsically beneficial rewards. The value of pursuing an action in a given state can be computed by the sum of all current and expected future rewards, discounted by the costs associated with taking the action. Optimizing this value function involves choosing actions that maximize this computation. This process of predicting an agent's future behavior on the basis of a set of rewards and constraints can be described as a Markov decision process (MDP)

The MDP framework captures the process by which agents use a model of their environment to decide which actions to take from its current state. As such, MDPs can represent a causal model for how an agent's actions change its environmental state on the basis of its subjective costs and rewards. For example, consider how a cleaning robot might navigate a room to pick up trash and avoid falling down the stairs (Figure 1). The agent (cleaning robot) decides which actions to take (e.g. move right) in order to maximize rewards it gains from picking up trash items located at the goal state, while avoiding states that return negative values, such as falling down a flight of stairs in the room. The robot's battery is depleted $(-1)$ by the energy required to move in any direction, so it must learn to optimize its behavior to efficiently reach its goals. 


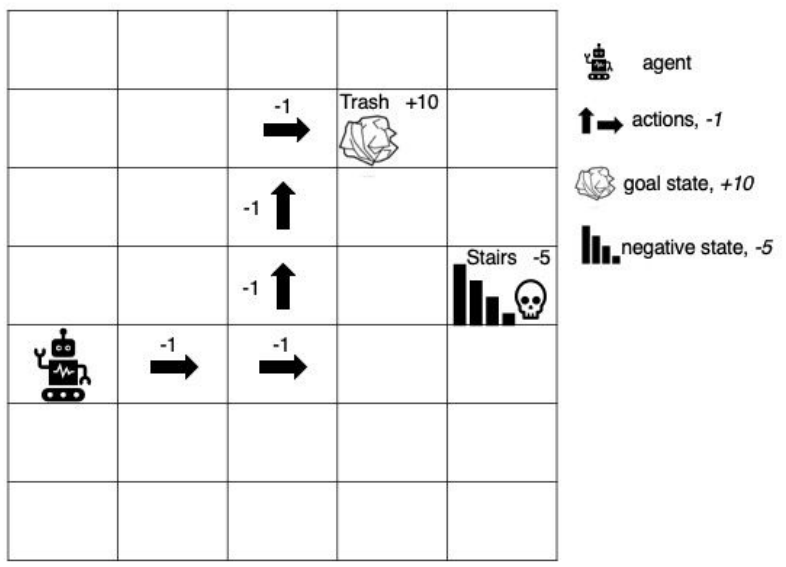

Figure 1. Example of Markov Decision Process

A criticism of using MDPs as a model of rational behavior, however, is that agents in the real world rarely possess full knowledge of their environment. For example, furniture or other items in the room may occlude the locations of trash rewards for the cleaning robot. Partially observable Markov decision processes (POMDPs) attempt to model the causal relationship between an agent's beliefs and their actions given their uncertainty about the state of the world (Smallwood \& Sondik, 1973; Sondik, 1978). Like MDPs, POMDPs attempt to find an optimal set of actions for an RL agent to maximize its reward. However, because the agent does not have full information about the world, the framework requires the ability to represent beliefs about the possible states of the world, which are updated based on the agent's observations. Thus, the expected future rewards from taking an action are based on the agent's internal beliefs about the state world.

POMDPs can provide a useful framework to model mentalizing. For example, Baker et al., (2017) use this framework to formulate a Bayesian theory-of-mind (TOM) model that attempts to infer an agent's likely goal given its uncertain beliefs about the state of the world. In their experiment, they create a 2D gridworld environment of a parking lot containing two of three possible food trucks. A solid wall occludes the agent's view of the full environment, so that it can only observe one truck from its starting state location. The authors represent the agent's observations in this spatial setting by indicating its visual line-of-sight, which allows the observer to know what information the agent possesses when making decisions. Participants are tasked with predicting the agent's beliefs and desires. The Bayesian TOM model attempts to capture an observer's hypothesis space of the agent's mind over the possible beliefs about which unseen food truck is on the other side of the wall, and the agent's possible reward function from simply observing the agent's observed actions. There are several components to the model. First, there is a term specifying the observer's prior belief $P\left(B_{t-1}, D, S\right)$ over the agent's initial beliefs $B_{t-1}$ (which truck is beyond the wall), desires $D$ (which truck the agent hopes is beyond the wall according to its preferences), and possible world state $S$ (their position in the grid). Second, there is a likelihood function capturing what the observer believes 
the agent can see (percept $P$ ) given their position in the grid $P(P \mid S)$. Third, there is a likelihood function capturing the observer's model of how the agent updates their beliefs about which truck is behind the wall based on their percept, $P\left(B_{t} \mid P, B_{t-1}\right)$. Finally, there is a likelihood function describing the observer's model of the agent's action plan $A$ given their beliefs of which truck is behind the wall and desires $P\left(A \mid B_{t}, D\right)$.

$$
P(B, D, P, S \mid A) \propto P\left(A \mid B_{t}, D\right) \times P\left(B_{t} \mid P, B_{t-1}\right) \times P(P \mid S) \times P\left(B_{t-1}, D, S\right)
$$

Across the space of possible beliefs and desires the agent could have, the observer evaluates the likelihood of generating the observed behavior given the hypothesized theory of the agent's mental state. Through a simple Bayesian update, the observer integrates this likelihood with the prior joint probability over mental states, yielding a posterior update that captures its inference of the agent's beliefs and desires (Baker et al., 2017; Baker, Saxe, \& Tenenbaum, 2009). Overall, the authors find strong evidence that this model accurately captures experimental participants' judgments about the agent's beliefs and desires across a range of environments. Importantly, models that selectively lesioned representations of the agent's beliefs, desires, or percepts were unable to accurately capture observers' judgments, suggesting that people were jointly considering all of this information.

\section{Summary}

Building on Lewin's early social psychology theory, which attempted to describe behavior as a function of a person operating within a specific environment, the fields of game theory and reinforcement learning have made substantial advances in the past several decades developing formal models of people and environments. In this chapter, we have briefly reviewed the innovations within these domains to provide a general framework to model the goals of an agent (e.g., maximize reward, or minimize embarrassment), modules for how they might represent the mental states of other agents, such as their beliefs, goals, desires, intentions, and feelings, and modules for how to integrate internal goals and mentalizing computations to produce optimal policies to navigate the environment. The strengths of this computational approach is that the framework and mathematical operationalization of these constructs facilitates collaborations across different laboratories and also scientific disciplines. Moreover, this framework is extensible and new modules can be added to an agent or further refined to generalize across more complex environments (Jolly \& Chang, 2019).

Though we believe computational approaches will aid in advancing our understanding of mentalizing, they are not without limitations, which are important to acknowledge. First, though there was early interest in integrating mathematical constructs into social psychological theory (Lewin, 1936, 1938), this vision never came into fruition as the field matured. Instead the disciplines that have made substantial advances in this area such as economics, cognitive science, and computer science have emphasized technical sophistication and incorporated 
advanced mathematical and computing training in both undergraduate and graduate training programs. Psychology will have to make significant concerted efforts to update their training curriculum to be able to continue to contribute to this interdisciplinary endeavor (Jolly \& Chang, 2019). Second, though the modeling is quite advanced, the mental operations and environments studied using this approach are necessarily oversimplified, which limits the sophistication of psychological inferences and generalizability of the findings. Most of the models discussed in this review are highly specific to a particular environment or game and it is unclear how well they will generalize to new contexts. Third, as is becoming clear with the rapid advances afforded by the powerful function approximations made possible by deep neural network architectures, there is a tradeoff between predictive power and model interpretability. State of the art models are now able to outperform the greatest strategic human minds at almost any type of complex game, but these models require enormous datasets, vast computational resources to train, and are not easily interpretable by humans. These deep learning approaches differ in their goals and as such, they do not necessarily provide direct insight into the computations underlying human mental operations. As these modeling endeavors begin to introduce similar cognitive and computational constraints as humans, they may begin to better reveal how humans are solving these problems. In summary, developing computational models of how humans perform mentalizing operations is an active and exciting area of research and much of its recent growth can be attributed to multidisciplinary efforts. 
Running Head: Modeling Mentalizing

\section{Acknowledgments}

This work was supported by funding from the National Institute of Mental Health R01MH116026 and R56MH080716 and the National Science Foundation CAREER 1848370. 


\section{References}

Abbeel, P., \& Ng, A. Y. (2004). Apprenticeship Learning via Inverse Reinforcement Learning. Proceedings of the Twenty-First International Conference on Machine Learning, 1 - . New York, NY, USA: ACM.

Achim, A. M., Guitton, M., Jackson, P. L., Boutin, A., \& Monetta, L. (2013). On what ground do we mentalize? Characteristics of current tasks and sources of information that contribute to mentalizing judgments. Psychological Assessment, 25(1), 117-126.

Akerlof, G. A. (1982). Labor contracts as partial gift exchange. The Quarterly Journal of Economics. Retrieved from https://academic.oup.com/qje/article-abstract/97/4/543/1846076

Amodio, D. M., \& Frith, C. D. (2006). Meeting of minds: the medial frontal cortex and social cognition. Nature Reviews. Neuroscience, 7(4), 268-277.

Baker, C. L., Jara-Ettinger, J., Saxe, R., \& Tenenbaum, J. B. (2017). Rational quantitative attribution of beliefs, desires and percepts in human mentalizing. Nature Human Behaviour, 1(4), s41562-017-0064.

Baker, C. L., Saxe, R., \& Tenenbaum, J. B. (2009). Action understanding as inverse planning. Cognition, 113(3), 329-349.

Baltaxe, C. A. (1991). Vocal communication of affect and its perception in three- to four-year-old children. Perceptual and Motor Skills, 72(3 Pt 2), 1187-1202.

Baron-Cohen, S., Leslie, A. M., \& Frith, U. (1985). Does the autistic child have a "theory of mind" ? Cognition, 21(1), 37-46.

Bartra, O., McGuire, J. T., \& Kable, J. W. (2013). The valuation system: a coordinate-based meta-analysis of BOLD fMRI experiments examining neural correlates of subjective value. Neurolmage, 76, 412-427.

Battigalli, P., \& Dufwenberg, M. (2007). Guilt in games. The American Economic Review, 97(2), 170-176.

Battigalli, P., \& Dufwenberg, M. (2009). Dynamic psychological games. Journal of Economic Theory, 144(1), 1-35.

Battigalli, P., Dufwenberg, M., \& Others. (2019). Psychological Game Theory. Retrieved from https://eller.arizona.edu/sites/default/files/Econ-WP-19-06.pdf

Battigalli, P., Dufwenberg, M., \& Smith, A. (2015). Frustration and Anger in Games. Retrieved from http://papers.ssrn.com/sol3/papers.cfm?abstract_id=2591839

Blakemore, S.-J. (2008). The social brain in adolescence. Nature Reviews. Neuroscience, 9(4), 267-277.

Bolton, G. E., \& Ockenfels, A. (2000). ERC: A Theory of Equity, Reciprocity, and Competition. The American Economic Review, 90(1), 166-193.

Brown, N., \& Sandholm, T. (2019). Superhuman Al for multiplayer poker. Science. https://doi.org/10.1126/science.aay2400

Burke, C. J., Tobler, P. N., Baddeley, M., \& Schultz, W. (2010). Neural mechanisms of observational learning. Proceedings of the National Academy of Sciences of the United States of America, 107(32), 14431-14436.

Call, J., \& Tomasello, M. (1999). A nonverbal false belief task: the performance of children and great apes. Child Development, 70(2), 381-395.

Camerer, C. F., Ho, T.-H., \& Chong, J. K. (2015). A psychological approach to strategic thinking in games. Current Opinion in Behavioral Sciences, 3, 157-162. 
Camerer, C., Ho, T., \& Chong, K. (2003). Models of Thinking, Learning, and Teaching in Games. The American Economic Review, 93(2), 192-195.

Campbell, M., Hoane, A. J., \& Hsu, F.-H. (2002). Deep Blue. Artificial Intelligence, 134(1), 57-83.

Chang, L. J., Doll, B. B., van 't Wout, M., Frank, M. J., \& Sanfey, A. G. (2010). Seeing is believing: trustworthiness as a dynamic belief. Cognitive Psychology, 61(2), 87-105.

Chang, L. J., \& Sanfey, A. G. (2013). Great expectations: neural computations underlying the use of social norms in decision-making. Social Cognitive and Affective Neuroscience, 8(3), 277-284.

Chang, L. J., \& Smith, A. (2015). Social emotions and psychological games. Current Opinion in Behavioral Sciences, 5, 133-140.

Chang, L. J., Smith, A., Dufwenberg, M., \& Sanfey, A. G. (2011). Triangulating the neural, psychological, and economic bases of guilt aversion. Neuron, 70(3), 560-572.

Cheong, J. H., Jolly, E., Sul, S., \& Chang, L. J. (2017). Computational Models in Social Neuroscience. Computational Models of Brain and Behavior, pp. 229-244. https://doi.org/10.1002/9781119159193.ch17

Chong, T. T.-J., Cunnington, R., Williams, M. A., Kanwisher, N., \& Mattingley, J. B. (2008). fMRI adaptation reveals mirror neurons in human inferior parietal cortex. Current Biology: $C B$, 18(20), 1576-1580.

Collette, S., Pauli, W. M., Bossaerts, P., \& O'Doherty, J. (2017). Neural computations underlying inverse reinforcement learning in the human brain. eLife, 6.

https://doi.org/10.7554/eLife.29718

Coricelli, G., \& Nagel, R. (2009). Neural correlates of depth of strategic reasoning in medial prefrontal cortex. Proceedings of the National Academy of Sciences of the United States of America, 106(23), 9163-9168.

Darwin, C. (1886). The Expression of the Emotions in Man and Animals.

Delgado, M. R., Frank, R. H., \& Phelps, E. A. (2005). Perceptions of moral character modulate the neural systems of reward during the trust game. Nature Neuroscience, $8(11)$, 1611-1618.

Dennett, D. C. (1978). Beliefs About Beliefs. The Behavioral and Brain Sciences, 1(4), 568.

Duchenne de Boulogne, G. B. A. (1876). Mécanisme de la Physionomie Humaine ou Analyse Électro-Physiologique de l'Expression des Passions. 2nd edn. Librairie J. B. Bailliere et Fils: Paris.

Falk, A., Fehr, E., \& Fischbacher, U. (2003). On the Nature of Fair Behavior. Economic Inquiry, 41(1), 20-26.

Fareri, D. S., Chang, L. J., \& Delgado, M. R. (2012). Effects of direct social experience on trust decisions and neural reward circuitry. Frontiers in Neuroscience, 6, 148.

Fehr, E., Bernhard, H., \& Rockenbach, B. (2008). Egalitarianism in young children. Nature, 454(7208), 1079-1083.

Fehr, E., \& Schmidt, K. M. (1999). A Theory of Fairness, Competition, and Cooperation. The Quarterly Journal of Economics, 114(3), 817-868.

Frith, C. D., \& Frith, U. (2006). The neural basis of mentalizing. Neuron, 50(4), 531-534.

Geanakoplos, J., Pearce, D., \& Stacchetti, E. (1989). Psychological games and sequential rationality. Games and Economic Behavior, 1(1), 60-79.

Goranson, R. E., \& Berkowitz, L. (1966). Reciprocity and responsibility reactions to prior help. Journal of Personality and Social Psychology, 3(2), 227-232. 
Hampton, A. N., Bossaerts, P., \& O'Doherty, J. P. (2008). Neural correlates of mentalizing-related computations during strategic interactions in humans. Proceedings of the National Academy of Sciences of the United States of America, 105(18), 6741-6746.

Heider, F., \& Simmel, M. (1944). An Experimental Study of Apparent Behavior. The American Journal of Psychology, 57(2), 243-259.

Hill, M. R., Boorman, E. D., \& Fried, I. (2016). Observational learning computations in neurons of the human anterior cingulate cortex. Nature Communications, 7, 12722.

Jara-Ettinger, J. (2019). Theory of mind as inverse reinforcement learning. Current Opinion in Behavioral Sciences, 29, 105-110.

Jenkins, A. C., Macrae, C. N., \& Mitchell, J. P. (2008). Repetition suppression of ventromedial prefrontal activity during judgments of self and others. Proceedings of the National Academy of Sciences of the United States of America, 105(11), 4507-4512.

Jern, A., Lucas, C. G., \& Kemp, C. (2017). People learn other people's preferences through inverse decision-making. Cognition, 168, 46-64.

Jolly, E., \& Chang, L. J. (2019). The Flatland Fallacy: Moving Beyond Low-Dimensional Thinking. Topics in Cognitive Science. Retrieved from https://onlinelibrary.wiley.com/doi/abs/10.1111/tops.12404

King-Casas, B., Tomlin, D., Anen, C., Camerer, C. F., Quartz, S. R., \& Montague, P. R. (2005). Getting to know you: reputation and trust in a two-person economic exchange. Science, 308(5718), 78-83.

Koster-Hale, J., \& Saxe, R. (2013). Theory of mind: a neural prediction problem. Neuron, 79(5), 836-848.

Krupenye, C., Kano, F., Hirata, S., Call, J., \& Tomasello, M. (2016). Great apes anticipate that other individuals will act according to false beliefs. Science, 354(6308), 110-114.

Lewin, K. (1936). Principles of topological psychology. https://doi.org/10.1037/10019-000

Lewin, K. (1938). The conceptual representation and the measurement of psychological forces. Retrieved from https://psycnet.apa.org/psycinfo/2008-10436-000/

Lindström, B., Golkar, A., Jangard, S., Tobler, P. N., \& Olsson, A. (2019). Social threat learning transfers to decision making in humans. Proceedings of the National Academy of Sciences of the United States of America. https://doi.org/10.1073/pnas.1810180116

McCabe, K. A., \& Smith, V. L. (2000). A comparison of naïve and sophisticated subject behavior with game theoretic predictions. Proceedings of the National Academy of Sciences, 97(7), 3777-3781.

McClure, S. M., Berns, G. S., \& Montague, P. R. (2003). Temporal prediction errors in a passive learning task activate human striatum. Neuron, 38(2), 339-346.

Mitchell, J. P., Heatherton, T. F., \& Macrae, C. N. (2002). Distinct neural systems subserve person and object knowledge. Proceedings of the National Academy of Sciences of the United States of America, 99(23), 15238-15243.

Mnih, V., Kavukcuoglu, K., Silver, D., Rusu, A. A., Veness, J., Bellemare, M. G., ... Hassabis, D. (2015). Human-level control through deep reinforcement learning. Nature, 518(7540), 529-533.

Moravčík, M., Schmid, M., Burch, N., Lisý, V., Morrill, D., Bard, N., ... Bowling, M. (2017). DeepStack: Expert-level artificial intelligence in heads-up no-limit poker. Science, 356(6337), 508-513.

Müri, R. M. (2016). Cortical control of facial expression. The Journal of Comparative Neurology, 524(8), 1578-1585. 
Nagel, R. (1998). A survey on experimental'beauty contest games': Bounded rationality and learning. Forthcoming in Games and Human Behavior: Essays in Honor of Amnon Rapoport eds. D. Budescu, I. Erev, and R. Zwick. Lawrence Erlbaum Associates: New Jersey.

Nash, J. F. (1950). Equilibrium Points in N-Person Games. Proceedings of the National Academy of Sciences of the United States of America, 36(1), 48-49.

Newell, A. (1994). Unified Theories of Cognition. Harvard University Press.

Newell, A. (2014). You can't play 20 questions with nature and win: Projective comments on the papers of this symposium. Retrieved from https://kilthub.cmu.edu/articles/You_can_t_play_20_questions_with_nature_and_win_proje ctive_comments_on_the_papers_of_this_symposium/6612977/files/12105638.pdf

Ng, A. Y., Russell, S. J., \& Others. (2000). Algorithms for inverse reinforcement learning. Icml, 1 , 2.

O'Doherty, J., Hampton, A., \& Kim, H. (2007). Model-Based fMRI and Its Application to Reward Learning and Decision Making. Annals of the New York Academy of Sciences, 1104(1), 35-53.

O'Doherty, J. P., Dayan, P., Friston, K., Critchley, H., \& Dolan, R. J. (2003). Temporal difference models and reward-related learning in the human brain. Neuron, 38(2), 329-337.

O'Reilly, R. C., Hazy, T. E., \& Herd, S. A. (2016). The Leabra Cognitive Architecture: How to Play 20 Principles with Nature. The Oxford Handbook of Cognitive Science, 91.

Pantelis, P. C., Byrge, L., Tyszka, J. M., Adolphs, R., \& Kennedy, D. P. (2015). A specific hypoactivation of right temporo-parietal junction/posterior superior temporal sulcus in response to socially awkward situations in autism. Social Cognitive and Affective Neuroscience, 10(10), 1348-1356.

Parkinson, C., Walker, T. T., Memmi, S., \& Wheatley, T. (2017). Emotions are understood from biological motion across remote cultures. Emotion , 17(3), 459-477.

Pearce, J. M., \& Hall, G. (1980). A model for Pavlovian learning: variations in the effectiveness of conditioned but not of unconditioned stimuli. Psychological Review, 87(6), 532-552.

Rabin, M. (1993). Incorporating Fairness into Game Theory and Economics. The American Economic Review, 83(5), 1281-1302.

Rescorla, R. A., Wagner, A. R., \& Others. (1972). A theory of Pavlovian conditioning: Variations in the effectiveness of reinforcement and nonreinforcement. Classical Conditioning II: Current Research and Theory, 2, 64-99.

Richardson, H., Lisandrelli, G., Riobueno-Naylor, A., \& Saxe, R. (2018). Development of the social brain from age three to twelve years. Nature Communications, 9(1), 1027.

Rosenthal, I. A., Hutcherson, C. A., Adolphs, R., \& Stanley, D. A. (2019). Deconstructing Theory-of-Mind Impairment in High-Functioning Adults with Autism. Current Biology, Vol. 29, pp. 513-519.e6. https://doi.org/10.1016/j.cub.2018.12.039

Sanfey, A. G., Rilling, J. K., Aronson, J. A., Nystrom, L. E., \& Cohen, J. D. (2003). The neural basis of economic decision-making in the Ultimatum Game. Science, 300(5626), 1755-1758.

Saxe, R., \& Kanwisher, N. (2003/8). People thinking about thinking people: The role of the temporo-parietal junction in "theory of mind." Neurolmage, 19(4), 1835-1842.

Selbing, I., \& Olsson, A. (2017). Beliefs about others' abilities alter learning from observation. Scientific Reports. Retrieved from https://www.nature.com/articles/s41598-017-16307-3

Silver, D., Hubert, T., Schrittwieser, J., Antonoglou, I., Lai, M., Guez, A., ... Hassabis, D. (2018). 
A general reinforcement learning algorithm that masters chess, shogi, and Go through self-play. Science, 362(6419), 1140-1144.

Simon, H. A. (1956). Rational choice and the structure of the environment. Psychological Review, 63(2), 129-138.

Smallwood, R. D., \& Sondik, E. J. (1973). The Optimal Control of Partially Observable Markov Processes over a Finite Horizon. Operations Research, 21(5), 1071-1088.

Sondik, E. J. (1978). The Optimal Control of Partially Observable Markov Processes over the Infinite Horizon: Discounted Costs. Operations Research, Vol. 26, pp. 282-304. https://doi.org/10.1287/opre.26.2.282

Stahl, D., \& Wilson, P. (1995). On Players' Models of Other Players: Theory and Experimental Evidence. Games and Economic Behavior, 10(1), 218-254.

Sul, S., Güroğlu, B., Crone, E. A., \& Chang, L. J. (2017). Medial prefrontal cortical thinning mediates shifts in other-regarding preferences during adolescence. Scientific Reports, 7(1), 8510.

Sutton, R. S., \& Barto, A. G. (1998). Introduction to reinforcement learning (Vol. 135). MIT press Cambridge.

Sutton, R. S., \& Barto, A. G. (2018). Reinforcement Learning: An Introduction. MIT Press.

Suzuki, S., Harasawa, N., Ueno, K., Gardner, J. L., Ichinohe, N., Haruno, M., ... Nakahara, H. (2012). Learning to simulate others' decisions. Neuron, 74(6), 1125-1137.

Tesauro, G. (1994). TD-Gammon, a Self-Teaching Backgammon Program, Achieves Master-Level Play. Neural Computation, 6(2), 215-219.

Trivers, R. L. (1971). The Evolution of Reciprocal Altruism. The Quarterly Review of Biology, 46(1), 35-57.

van Baar, J. M., Chang, L. J., \& Sanfey, A. G. (2019). The computational and neural substrates of moral strategies in social decision-making. Nature Communications, 10(1), 1483.

Van Overwalle, F. (2009). Social cognition and the brain: a meta-analysis. Human Brain Mapping, 30(3), 829-858.

von Neumann, J., \& Morgenstern, O. (2007). Theory of Games and Economic Behavior. Princeton University Press.

Wellman, H. M., Cross, D., \& Watson, J. (2001). Meta-analysis of theory-of-mind development: The truth about false belief. Child Development, 72(3), 655-684.

Wimmer, H., \& Perner, J. (1983). Beliefs about beliefs: representation and constraining function of wrong beliefs in young children's understanding of deception. Cognition, 13(1), 103-128.

Yoshida, W., Dolan, R. J., \& Friston, K. J. (2008). Game theory of mind. PLoS Computational Biology, 4(12), e1000254.

Yoshida, W., Seymour, B., Friston, K. J., \& Dolan, R. J. (2010). Neural mechanisms of belief inference during cooperative games. The Journal of Neuroscience: The Official Journal of the Society for Neuroscience, 30(32), 10744-10751. 